\title{
THE TREATMENT OF DUPUYTREN'S CONTRACTURE
}

\author{
Mortimer H. Shaw, B.Sc. M.B., Ch.B., F.R.C.S. \\ Plastic Surgeon, St. Fames's Hospital, Leeds
}

Although Sir Astley Cooper gave a clinical description of this condition in 1822 in this country, it was Baron Guillaume Dupuytren ten years later in Paris, who identified the nature of the disorder by dissection as a contracture of the palmar fascia, and suggested fasciotomy as a method of treatment (1832). The cause of the disorder remains unknown, but it seems certain that a hereditary predisposition is present in about $12 \%$ of patients. Some believe that single blows or repeated small injuries may initiate the fibrosis in the palmar fascia in susceptible individuals; but all hands are liable to trauma, and comparable series of cases occurring in manual and nonmanual occupations have shown similar incidence. The condition affects men ten times as often as women; is common in white-skinned people of Western countries and rare in those of dark-skin. The reason for this is not apparent. It is essentially a bilateral disease but there is often a delay of years before the onset in the second hand. The clinical progress is extremely variable, the disorder being present without causing any disability at all in many hands throughout adult years; but it may at any time produce disability by contracture, without demonstrable cause. The disability most often calls for relief in men between the ages of 40 and 65 . There is, however, a small group of patients in whom the contracture arises at an earlier age, is more prone to recur after treatment, and in whom similar fibrous deposits are found on the dorsal aspect of the proximal interphalangeal joints (knuckle pads or Garrod's nodes), and in the plantar fascia and in whom a positive family history of a similar disorder may be obtainable (Hueston, 196r).

\section{Clinical Course}

It is convenient to adopt a simple classification of the clinical stages of the disorder as a guide to treatment.

The one we adopt is as follows:

Stage 1. A nodule present in the palmar fascia but without finger contracture or skin infiltration.

Stage 2. A nodule in the palm infiltrating the skin but without finger contracture.
Stage 3. A nodule with skin involvement and flexion contracture of one or more digits.

Stage 4. Flexion deformity with adhesions in tendon sheaths or joint capsules, or with subluxation.

In stage 1 , explanation and observation of the progress of the lesion is all that is necessary.

In stage 2 , the same applies unless skin involvement becomes widespread.

In stage 3 , excision of the contracted fascia is required to relieve the deformity before it becomes fixed (the extent of the excision will be discussed later).

In stage 4, amputation of a digit may be necessary in addition to fasciectomy or fasciotomy. However, it must never be assumed that the sacrifice of a finger is inevitable unless this is proved at operation, since a useful range of movement may often be obtained from an apparently fixed flexion deformity of the little finger once the bands have been resected.

\section{Treatment}

Some patients seek advice because the flexion of the fingers is a nuisance, or threatens to become so, in their daily activities. Others because they are alarmed to find an unexplained lump in the palm. Surgical treatment is directed to the relief of the contracture by the method most suitable for the individual patient according to his needs and constitution. The method chosen should cause the least disturbance of the physiology of the hand consistent with improvement of the deformity and a reasonable expectation of freedom from recurrence. Any hand operation may give disappointing results unless a precise technique is employed in a bloodless field.

Strict post-operative observation and care is imperative to avoid or treat complications, the most troublesome of which in order of frequency are palmar hæmatoma, persistent œdema, loss of skin or delay in healing of the palm. All these complications may result in prolonged and even permanent loss of full finger flexion, weakness of grip, loss of suppleness in the hand and lack of rotational movement around the middle finger 
metacarpal. In addition the hand may show circulatory changes and be blue, cold and uncomfortable. Lack of full extension of the fingers by residual or recurrent contracture is of small significance compared with the loss of full finger flexion which may follow such complications, or may result from a badly executed or ill-chosen operation. A selective approach is therefore advisable depending upon the following factors.

1. The type of discase and extcnt of involvement of the hand.

2. Family history.

3. The age of the pasient.

4. Concomitant disorders such as cardiovascular disease.

5. Int $\epsilon$ lligence and co-operation of the patient.

6. Constitutional tcndency to swell.

7. Occupation and manual dexterity.

8. Tendency to small joint arthritis.

9. Experience of the opcrator.

Io. Quality of after care.

A choice can be made on the lines suggested by Julian Bruner (1960) between:-

I. No treatment at all.

2. Primary amputation of a finger.

3. Simple fasciotomy.

4. Limited fasciectomy.

5. Wide fasciectomy.

\section{No Treatment}

In early disease without disability periodical inspection and encouragement is all that is needed. In late contractures in retired and elderly people the condition may also occasionally remain static without treatment.

\section{Primary Amputation of a Finger}

In cases with secondary changes in tendon and joints and in those where recurrence of the contracture is accompanied by widespread skin change, poor circulation and diffuse scarring in the fingers, amputation may be the quickest way to restore function without putting the rest of the hand to the risk of damage by the complication of hæmatoma or œdema.

\section{Simple Fasciotomy}

The simple subcutaneous division of a localized palmar fascial band may give temporary and in some cases lasting relief, and restore use to a finger. The method is indicated in the aged and infirm and may be all that is required in those rather localized strong bands in which the active contractive phase is over and in whom joints and tendon sheaths have not yet become stiff by secondary change. The method is unsuitable in the finger because of the possibility of damage to digital nerves. In diffuse involvement in the palm and in young patients the method is too often followed by recurrence of the contracture withif a short time to be confidently recommended, and sometimes multiple fasciotomy wounds make dissection of the palm more difficult on a subse $\mathbb{D}$ quent occasion.

\section{Limited Fasciectomy}

This operation is designed to remove only the fascia which can be seen by the naked eye to be्en the cause of the deformity, together with a smalf margin of normal fascia on each side. Fasciectomy is a block removal of the involved fascia, and nots simply an open fasciotomy. Compared with wides fasciectomy (discussed below) the hand is healed. more quickly and the patient may return to workw a little sooner. There is a lower risk of hæmatomao This is the operation of choice when the bands? are clear-cut and localized, and when the skin of. the palm is not widely involved. It should be used in preference to the wider dissection if there is ants tendency to arthritis of the small finger joints, of the constitution of the subject is such as mighẹ predispose to post-operative swelling. It should be employed in the older patients, and in those who exhibit the more severe form of Dupuytren diathesis associated with knuckle pads and earlie onset. Recurrence and extension in the palm wilt take place more often than it will after a wi\&eo dissection; secondary operation is likely to required more frequently, but the complications which follow this limited operation are less fres quent and less serious. The incision is a longitudio nal one over the contracting band in the palm and involved finger. Extreme care is taken to avoi damage to the subdermal plexus of vessels. Th $\vec{E}$ palmar incision may be made 'zig-zag' in form to minimize linear contracture of the subsequen scar, if lengthening of the skin in the long axis is. not necessary. Lengthening, however, is commonly required in the finger, and is conveniently accom? plished by means of a $\mathrm{Z}$-plastic, at the expense of shortening in the transverse axis. A Z-shapeç incision with three equal limbs, each enclosing angles of $60^{\circ}$, outline two flaps, which are transposed at the time of closure of the wound.

Careful hæmostasis is necessary before closure of the wound. Several methods are in common. use to avoid hæmatoma. These include simples drainage, suction drainage, gauze and crêpê bandage compression, and plaster slabs. NQ method is infallible. Elevation of the hand in the post-operative hours is helpful in minimizing swelling. Early exposure of the hand and fingerso with the stitches still in position, is a help in the encouragement of early restoration of finger movements. The hand is returned gradually to normas activity. Special exercises and routine physio $\vec{D}$ therapy are not necessary, but the physiotherapisfo 
may encourage normal use of the hand and shoulder, which helps to prevent œdema.

\section{Wide Dissection}

A wide excision of the palmar fascia through a transverse incision in the palm has been recommended by McIndoe and Beare (1958), Skoog (1948), and Shaw (1952). It is indicated when the involvement of the palm is diffuse and wide in fit middle-aged men. It should be performed by surgeons skilled in hand surgery and with good facilities for after-care and with the full knowledge that the complications following the operation are more common than following the limited dissection. If a hæmatoma occurs and remains in the hand, organization to fibrous tissue will produce diffuse scar, and will limit active movement of the finger joints and give rise to odema. The rise in tension may produce loss of skin which will need grafting; and the delay in healing will be sufficient to postpone the return of the hand to its normal use. Finger stiffness almost inevitably follows a hæmatoma which is neglected. On the other hand if the hæmatoma is recognized as such and is completely evacuated by operation in the theatre in 24 to 48 hours, and its return is prevented by suitable treatment, normal function can still follow. Continuous post-operative negative pressure in the dead space produced by the wide dissection (McFarlane, r959) has been found helpful in reducing the tendency to hæmatoma, promoting early healing and restoration of finger movements. By this means the hand can be observed repeatedly in the early post-operative hours for hæmatoma, while the skin remains dry and cool. The relative positive pressure so formed is localized to the palm of the hand, while lateral and dorsal compression are avoided. A few of the cases complicated by hæmatoma will be followed by loss of skin requiring grafting but if the loss of skin is recognized early and made good by grafting, once again a good functional result can follow, although deficient sensation in the grafted skin is a handicap. If delayed healing due to loss of skin is accepted, some stiffness of the fingers can be expected to take place which may last for some months. The radical fasciectomy is contraindicated in the younger patient with a family history of the condition and in whom there is a constitutional tendency to recurrence even after a wide removal. It is unwise in people of bulky stature with thickened skin folds who may be constitutionally prone to post-operative swelling. (Barclay, 1959). It is unnecessary in the elderly infirm who may not live long enough to see its benefit. The results are not so satisfactory in women and the operation should be studiously avoided in the presence of arthritic joints. Following successful wide dissection of the fascia, however, consistently good results can be obtained with a greater period of freedom from a local recurrence and a prospect of fewer secondary operations.

Further research is needed to elucidate the cause of the contracture, and allow a more scientific approach to prevention and treatment of this common disability.

\section{REFERENCES}

Barclay, T. L. (1959): OEdema Following Operation for Dupuytren's Contracture, Plast. reconstr. Surg., $23,348$.

Bruner, Julian M. (1960): The Selective Treatment of Dupuytren's Contracture with Special Reference to Complications and Indications for Treatment, Transactions of the International Society of Plastic Surgery, p. 244.

COOPER, AstLEy (1822): 'A Treatise on Dislocations and on Fractures of the Joints', p. 524. London: John Churchill. DupuYtren, G. (1832): 'Leçons orales de clinique chirurgicale faites à L'Hotel Dieu de Paris', Vol. 1. Paris: Baillière.

Hueston, J. T. (196I): Limited Fasciectomy for Dupuytren's Contracture, Plast. reconstr. Surg., $27,569$.

McFarlane, R. M. (1959): The Use of Continuous Suction after Operation for Dupuytren's Contracture, Brit. $\mathcal{~}$. plast. Surg., II, 301.

McINDOE, A. H., and BeAre, R. L. B. (1958): The Surgical Management of Dupuytren's Contracture, Amer. F. Surg., 95, 197.

Shaw, M. H. (1952): Treatment of Dupuytren's Contracture, Brit. F. Plast. Surg., 4, 218.

and BARCLAY, T. L. (1957): Dupuytren's Contracture. Results of Radical Fasciectomy, Transactions of the International Society of Plastic Surgeons, ist Conference, 1955, p. 428. Baltimore: Williams \& Wilkins.

Skoog, T. (1948): Dupuytren's Contraction, with Special Reference to Ætiology and Improved Surgical Treatment; Its Occurrence in Epileptics and Note on Knuckle Pads, Acta chir. scand., 96, suppl. 139. 